\section{Cahiers de Narratologie}

Analyse et théorie narratives

$28 \mid 2015$

Le récit comme acte cognitif

\title{
Les fonctions argumentatives des récits de vie : un exemple de construction ethotique chez Laurent Gbagbo
}

Dorgelès Houessou

\section{(2) OpenEdition}

Journals

Electronic version

URL: https://journals.openedition.org/narratologie/7227

DOI: 10.4000/narratologie. 7227

ISSN: 1765-307X

Publisher

LIRCES

\section{Electronic reference}

Dorgelès Houessou, "Les fonctions argumentatives des récits de vie : un exemple de construction ethotique chez Laurent Gbagbo", Cahiers de Narratologie [Online], 28 | 2015, Online since 29 October 2015, connection on 11 June 2021. URL: http://journals.openedition.org/narratologie/7227 ; DOI: https://doi.org/10.4000/narratologie.7227

This text was automatically generated on 11 June 2021 .

Article L.111-1 du Code de la propriété intellectuelle. 


\title{
Les fonctions argumentatives des récits de vie : un exemple de construction ethotique chez Laurent Gbagbo
}

\author{
Dorgelès Houessou
}

\section{Introduction}

1 L'arrière plan méthodologique général de ce travail est l'analyse du discours. Nous tenterons dans cette contribution de montrer à partir d'une étude de cas comment la narration peut s'inscrire dans les schèmes cognitifs de la manipulation lorsqu'elle est enchâssée dans un appareil discursif macro-structurel. Une telle configuration offre en termes d'efficacité un avantage certain au locuteur-narrateur dont le discours - le récit y compris - a des chances d'aboutir perlocutoirement. Nous nous intéresserons uniquement aux passages narrés en vue de déceler non seulement leur structure, mais aussi et surtout les modalités, de même que les enjeux de leur impact cognitif.

\section{Notes préliminaires sur le didactisme du genre narratif}

2 Le type narratif est quasi ontologiquement associé au souvenir de faits passés et à son élocution. Cette fonction de témoignage en est définitoire dans la plupart des occurrences y compris dans le cas du présent de narration, où le destinataire est contraint de saisir la simultanéité des faits et de la narration comme indice de réactualisation d'évènements anciens. Le cas spécifique de l'emploi prophétique du récit ne déroge pas à la règle. Ainsi, même en construisant une succession d'évènements censés être encore non actualisés, le récit prophétique n'en est pas moins associé au passé, car le narrateur du genre concerné relate un déjà vu. Il est de ce fait un témoin hors du temps car issu d'un monde présent où le futur est déjà passé 
puisqu'il en a fait l'expérience. C'est donc l'usage du passé qui dans ce cas tient lieu de certitude quant à la conformité des faits déjà vécus par le narrateur avec ceux à venir. De ce lien indéfectible avec le passé semble découler un nécessaire tri, une tendance psychique dominée par le choix de ne retenir pour soi et autrui que les épisodes de l'existence jugés dignes d'intérêt, c'est-à-dire les circonstances heureuses ou malheureuses dont la narration est utile aussi bien dans les perspectives psychologiques et morales que sociologiques et politiques. Une telle obsession du bien survit même dans les récits tragiques où le malheur et les drames présentés ont vocation à susciter l'attitude appropriée chez le destinataire si celui-ci était confronté aux mêmes épreuves. Il en résulte un didactisme par l'exemple incarné par le héros et un autre par le contre-exemple incarné par son double antinomique, l'antihéros. C'est tout l'enjeu du mythe que d'avoir, tout au long des siècles, favorisé l'apprentissage du monde à travers la représentation narrée des faits fondateurs d'un habitus (Danblon 2003) :

Chez les pré-hominidés, la fonction narrative aurait pris le relais lorsque les contraintes biologiques se sont estompées. Un ancien rapportait au sein du groupe le souvenir d'un événement traumatisant à l'origine d'une crise sociale mémorable afin de permettre à la communauté de les éviter. Les premières sociétés orales auraient trouvé, dans de telles habitudes discursives, une manière de jurisprudence qui puisait sa topique propre dans la mémoire orale des anciens. Les grandes histoires mythiques ont peut-être émergé à partir de ces premières habitudes narratives.

3 L'on comprendra mieux la portée sociologique des contes - formes profanes et éclatées des mythes - à la lumière de cette fonction didactique du type narratif, dont les mécanismes de captation et de persuasion sont constitutifs des fondamentaux mêmes du type concerné. Les exemples de la parabole en milieu religieux ou encore de la fable littéraire sont assez illustratifs de cette dynamique de la narration en tant que forme privilégiée de l'enseignement non seulement didactique, mais aussi moral. De ce fait toute narration est une énonciation satisfaisant au critère de l'efficacité, au sens austinien du terme, dans la mesure où le récit peut être une forme édulcorée de l'argumentation et que cette dernière n'est jamais neutre, comme le constate Searle (1969: 62) :

si l'on considère la notion d'acte illocutionnaire, il faut aussi considérer les conséquences, les effets que de tels actes ont sur les actions, les pensées ou les croyances, etc., des auditeurs. Par exemple, si je soutiens un argument je peux persuader, ou convaincre mon interlocuteur: si je l'avertis de quelque chose, je peux l'amener à faire ce que je lui demande; si je lui fournis une information je peux le convaincre, l'éclairer, l'édifier, l'inspirer, lui faire prendre conscience. Les expressions notées en italiques ci-dessus désignent des actes perlocutionnaires.

Ces actes perlocutionnaires sont davantage lestés d'un arrière plan axiologique dans le cas du récit discursivement encadré. Le discours politique est ainsi l'un des plus riches de ce recours au récit en vue de parvenir à ses fins de manipulation. Le corpus analysé ici est un discours de Laurent Gbagbo, Président de la Côte d'Ivoire de 2000 à 2011, prononcé lors de la cérémonie d'investiture de la mutuelle « Gotiwa » à Dribouo, le 12 septembre 2009. 


\section{De la manipulation}

5 Le concept de manipulation remonte certainement aux origines des premières investigations linguistiques. Platon y fait allusion lorsqu'il propose de discriminer le beau, dont l'objet est purement rhétorique, du vrai, résultant pour sa part d'une quête exclusivement épistémologique. Pour lui la beauté du style n'est qu'un artefact destiné à tromper l'auditoire quant à la teneur du discours, même lorsque celle-ci est viciée ${ }^{1}$. Aristote a également observé cette tension entre le beau et le vrai dans son approche du langage en ses déclinaisons poïétique et utilitaire. L'évincement de la vérité au bénéfice de la sublimation du vraisemblable dont les sophistes ont fait leur principe témoigne sans ambages de la ténacité de la question à travers les âges. Il en est résulté une ambivalence axiologique de la pratique de la rhétorique car si, comme Platon le pense, elle peut sembler hostile à la vérité dont la science seule peut percer les mystères, la rhétorique ne dispose pas moins d'un double angélique qui la fait prospérer en instrument de choix pour démasquer les faussetés, paralogismes et arguties qui participent d'une intention de manipulation ${ }^{2}$. La notion d'éthique est donc au cœur de toute approche de la manipulation. En cela elle est jugée pernicieuse à bien des égards.

6 Premièrement, la manipulation se définit en tant qu'action sur autrui (Salavastru 2002):

En un sens très large, manipulation signifie une action d'un individu (action soit de nature mécanique ou manuelle, soit de nature discursive) par l'intermédiaire de laquelle il peut modifier des choses, des états de choses, des actions, des sentiments, des croyances d'autres individus sans l'accord de ces derniers et, parfois, sans qu'ils le sachent.

7 Deuxièmement, cette action se caractérise par l'intentionnalité négative et occulte du locuteur, car il n'y a guère de manipulation lorsque le récepteur est conscient de l'intention du locuteur. Si tel était le cas il n'y aurait pas manipulation, mais conviction. C'est dans la mesure où l'auditoire est ignorant de l'intention manipulatrice que celle-ci aboutit perlocutoirement (Akopova 2013) :

La manipulation est réalisée lorsque l'auditeur ne peut pas voir les intentions cachées de l'orateur derrière ce qui est réellement dit. Comme l'un des principaux paramètres de l'énonciation manipulatrice est l'intentionnalité spécifique, afin de distinguer la manipulation, il faut analyser des paramètres tels que le but de la communication verbale, l'intention communicative, la raison, et le motif de celle-ci. La manipulation est un aspect pragmatique qui atteint ses objectifs sans la détection évidente de l'intention communicative : l'orateur choisit consciemment la forme d'énonciation qui dissimule le mieux son état intentionnel. En augmentant le niveau de perception erronée que les auditeurs ont du domaine de l'information, la manipulation s'élargit à la réalité subjective illusoire ${ }^{3}$.

8 Troisièmement, la manipulation implique une faiblesse d'esprit de la part des manipulés. Ceux-ci manquent d'esprit critique et ne disposent pas des «connaissances spécifiques qui pourraient être utilisées pour résister à la manipulation " (Van Dijk 2006). Ce qui précisément implique non plus un rapport énonciatif parallèle entre les acteurs, mais plutôt un lien de verticalité assuré par la position d'ascendance psychologique dont bénéficie le manipulateur. Quoique cette ascendance puisse tirer son origine d'une plus-value d'ordre physiologique (critère d'âge), social (critère institutionnel et de classe sociale), cognitif (critère d'instruction), spirituel (critère de 
croyance) etc., elle est d'autant plus aboutie lorsqu'elle combine plusieurs de ces domaines d'influence, comme cela est souvent le cas en politique.

9 Quatrièmement, la manipulation reproduit les schèmes de l'inégalité sociale. En tant qu'instrument d'abus de pouvoir ou d'ascendance, elle est antinomique à l'idéal démocratique auquel aspirent les sociétés modernes:

La manipulation implique non seulement le pouvoir, mais spécifiquement l'abus du pouvoir, c'est-à-dire la domination. Plus précisément, la manipulation implique l'exercice d'une forme d'influence illégitime au moyen du discours: les manipulateurs font croire aux autres ou leur font faire des choses qui sont dans l'intérêt du manipulateur, et contre celui des personnes manipulées ${ }^{4}$ (Van Dijk 2006).

Ce quatrième principe définitoire de la manipulation aboutit au cinquième qui est celui de la dangerosité de cette pratique pour les auditeurs et pour la stabilité sociale, ceci dans la mesure où elle ne sert que les intérêts égoïstes des manipulateurs.

la manipulation est illégitime dans une société démocratique, car elle (re)produit, ou peut reproduire l'inégalité : elle est accomplie aux seules fins de rechercher les intérêts supérieurs des groupes d'influence et des orateurs puissants, et nuit aux intérêts des groupes et orateurs les moins puissants [...] Les critères essentiels de la démarche manipulatrice sont que les récepteurs sont mûs au mépris de leur conscience, de leur volonté, de leurs intérêts, et que la manipulation est réalisée pour le seul bénéfice et les avantages exclusifs du manipulateur ${ }^{5}$ (Van Dijk 2006)

11 L'on retiendra donc avec Akopova (2013) que : «La manipulation est un phénomène psychologique et social dont l'exercice a des effets destructeurs aussi bien sur l'individu que sur la société dans son ensemble ${ }^{6}$ \%.

12 Le cadre particulier du discours politique constitue l'un des lieux les plus communs à la manipulation, vu qu'un locuteur ambitionne de modifier les croyances et les représentations mentales de ses auditeurs en vue de les faire agir conformément à sa volonté sans que ceux-ci ne s'en aperçoivent concrètement. Il s'agit de susciter de la sympathie ou de la consolider, et de pousser l'auditoire d'une part à manifester ce sentiment par l'adhésion sans restriction aux thèses du sujet discursif, et d'autre part à convertir ce capital de sympathie en suffrage pratique. Le corpus ici analysé recèle de stratégies discursives poursuivant un tel but. Nous nous intéressons particulièrement à celles qui découlent du recours constant à la narration dont le philosophe et rhéteur du IIIème siècle Longin (1995) notait déjà en ces termes la valeur de manipulation :

Ces Images, que d'autres appellent Peintures, ou Fictions, sont aussi d'un grand artifice pour donner du poids, de la magnificence, et de la force au discours. Ce mot d'Image se prend en général pour toute pensée propre à produire une expression, et qui fait une peinture à l'esprit de quelque manière que ce soit. Mais il se prend encore dans un sens plus particulier et plus resserré, pour ces discours que l'on fait, lorsque par un enthousiasme et un mouvement extraordinaire de l'âme, il semble que nous voyons les choses dont nous parlons, et quand nous les mettons devant les yeux de ceux qui écoutent.

Le cas des récits présents dans le corpus étudié est tout de même spécifique car il s'agit d'histoires autobiographiques, dont Sadowsky et Roche ont pu dire : «De par sa nature même enfin, la narration personnelle peut être dite "révisionniste". Les histoires autobiographiques étant des reconstructions plus que des récits complets et exacts d'évènements. » (John Sadowsky \& Loïck Roche 2010 : 143). De ce fait la narration n'est presque jamais une retranscription fidèle, car autant le traducteur est traitre, autant le narrateur réinvente, par la réappropriation axiologique qu'il en fait, les récits qu'il 
expose à un public donné. Ainsi, lors de la narration «les exigences de l'exactitude et de la vérité sont assouplies au profit des arguments. Les histoires sont des récits chargés d'émotions et de symboles, elles ne présentent pas des faits mais elles enrichissent, embellissent et donnent un sens, a posteriori, à des faits » (John Sadowsky \& Loïck Roche 2010 : 143-144). Plus incisif, Christian Salmon estime que la narration en situation discursive est un instrument systématique de manipulation, voire de propagande quand il s'agit d'enjeux politiques. Il cite d'ailleurs Lori Silverman, dont il partage le radicalisme, qui soutient que « les histoires sont comme des virus, elles sont contagieuses ». (Salmon $2007: 27)$ Berut considère dans une optique semblable que le récit est un puissant instrument de manipulation, voire de propagande (Berut 2010).

\section{Structure narrative des récits de vie}

14 Les récits de vie relevés dans notre corpus se subdivisent en deux formes principales, qui sont le récit de soi et le récit de l'autre. Chacune de ces formes adopte une structure énonciative différente. Nous nous intéresserons ici à la fois à l'énonciation et à la question du point de vue (PDV) «à partir de sa nature radicalement polyphonique, permettant au locuteur d'exprimer son PDV, ou de se positionner face aux PDV de ses interlocuteurs ou des tiers délocutés, tels qu'il les reconstruit dans son propre discours » (Rabatel 2003).

Aussi, envisager comme nous nous le proposons un implicite argumentatif au récit de vie, revient-il à reconsidérer les implications du point de vue dans l'argumentation, car «[...] par la dimension gnomique couplée à l'apparition des points de vue, l'argumentation se profile derrière la narration: simultanément petite histoire exemplaire à caractère proverbial et topique à caractère pré-inférentiel » (Danblon 2003).

\section{Le récit de soi}

16 Il se construit à partir de l'énonciation élocutive. Celle-ci, selon Charaudeau: "s'exprime à l'aide des pronoms personnels de la première personne accompagnée de verbes de modalité, d'adverbes et de qualificatifs qui révèlent l'implication de l'orateur et décrivent son point de vue personnel» (Charaudeau $2005: 135$ ). Il s'agit donc d'une énonciation autocentrée où le sujet locutif évoque des épisodes de sa vie et de son expérience antérieure au moment de l'énonciation. C'est le cas lorsque le locuteurnarrateur déclare :

C'était le 14 juillet 1990, à Bonoua. C'était pendant la période enflammée, dure. Nous venions de Korhogo où nous avions essuyé des coups et des quolibets. Nous sommes allés à Bonoua, où, à peine suis-je monté sur le podium pour délivrer mon message, que le Sous-Préfet en poste à l'époque dans cette localité a donné l'ordre de lancer sur moi des bombes lacrymogènes [...] Donc, quand on a envoyé en ma direction des bombes lacrymogènes et qu'on en a balancé sur la foule venue m'écouter, celle-ci s'est dispersée. C'étaient des cris et des hurlements; les gens fuyaient dans tous les sens. C'est dans ce contexte qu'une voiture de marque "Mercedes", d'un ami s'est approchée de moi. C'est ainsi que les trois personnes que j'ai citées plus haut, Guédé, Kobri et Akré, m'ont fait monter dans cette voiture pour assurer ma protection [...] Après avoir pris soin de sécuriser la route, ils m'ont ramené sur Abidjan. Le lendemain, c'est-à-dire le 15 juillet 1990, ils étaient encore à mes côtés, à Agboville. Pendant tout le trajet d'Abidjan à Agboville, un hélicoptère 
de l'Armée ivoirienne survolait ma voiture. Évidemment, dans cette ville, l'on avait cassé nos bâches que les militants avaient dressées. Nous avons été obligés de faire notre meeting au balcon d'un professeur, surnommé "John Jay", que j'ai d'ailleurs retrouvé, récemment.

D’Agboville, nous nous sommes rendus à Grand-Morié [Ndlr : village de feu Ernest Boka, premier Président de la Cour Suprême de Côte d'Ivoire]. Dans cette localité, nous avons trouvé des gendarmes pré-positionnés dans tous les coins du cimetière. De fait, l'on avait dit au Président Houphouet-Boigny [Ndlr : le premier Président de la République de Côte d'Ivoire] que je me rendais à Grand-Morié pour m'incliner sur la tombe de feu Ernest Boka ; ceci, dans le but d'accuser le Président HouphouetBoigny (de la mort de ce dernier, ndlr). Mais, je ne connaissais pas feu Ernest Boka. Je n'ai entendu parler de lui que dans les livres. J'allais donc faire un meeting à Grand-Morié parce qu'on m'y avait invité. Bref, c'est dans ces conditions que j'ai fait la connaissance du Capitaine Guédé. Donc, chaque fois qu'il s'agit d'aller à un évènement ou une invitation, chez Guédé, Kobri Rémi ou chez Akré Lucien, je suis là. Quand je suis empêché, je leur demande de reporter la date de l'évènement ou de l'invitation, pour me permettre d'être disponible. Parce que dans la vie, quand tu es ingrat, c'est Dieu lui-même qui te frappe. Mais la gratitude, ce n'est pas seulement une affaire d'argent [...] Tout sourire, un verre d'eau, mérite la gratitude.

(Laurent Gbagbo, samedi 12 septembre 2009)

Ce premier récit de soi est encadré selon les contraintes du genre (date, lieu, personnages, péripéties...). Ces précisions en font un témoignage, une histoire vraie qui, quoiqu'elle semble cocasse, est destinée pour le locuteur à révéler combien périlleux aura été son parcours d'unique opposant au premier président de la République ivoirienne. Des marqueurs axiologiques situent le contexte. Il s'agit donc d'une: "période enflammée, dure" au point où il subissait "des coups et des quolibets ». Il évoque ainsi trois étapes, trois villes que sont Bonoua, Agboville et Grand-Morié où il avait été invité pour des meetings. La diversité des localités citées contraste avec l'unicité du scénario, car à chacun de ces endroits il est confronté à l'appareil répressif de l'État qui use d'intimidation et de violence pour l'empêcher de s'exprimer. En effet, le locuteur-narrateur évoque à la fois la police, l'armée et la gendarmerie pour rappeler la violence à la fois psychologique et physique qu'il a endurée dans sa quête pour la démocratie. C'est tout de même avec pudeur qu'il accuse ces derniers à travers l'usage de l'indéfini pour décrire leurs actes « on a envoyé en ma direction des bombes lacrymogènes et [...] on en a balancé sur la foule venue m'écouter [...] l'on avait cassé nos bâches ». Une telle précaution énonciative laisse supposer que la visée perlocutoire de son récit n'est pas tant de porter un regard accusateur sur l'ancien chef d'État que de montrer sa détermination et son courage politique. En effet, l'énonciation étant proche de la campagne électorale de 2010, Laurent Gbagbo est précautionneux sur la nécessité de ne pas salir la mémoire du père de la nation dont ses adversaires se disent héritiers. L'« houphouetisme » est alors un concept érigé au rang de valeur nationale. D'où l'usage de l'indéfini visant à blanchir ce dernier, qui aurait été notamment manipulé par cet obscur ennemi en vue de susciter le courroux du président à l'endroit du locuteur-narrateur : «l'on avait dit au Président HouphouetBoigny que je me rendais à Grand-Morié pour m'incliner sur la tombe de feu Ernest Boka ; ceci, dans le but d'accuser le Président Houphouet-Boigny ». Ce procédé enrichit encore l'ethos du locuteur-narrateur, qui donne ainsi de lui l'image d'un homme assez bon pour pardonner les persécutions qu'il a systématiquement endurées en tant qu'opposant. Ce récit poursuit donc un double objectif perlocutoire. L'un, implicite, vise à persuader l'auditoire de l'attachement de Laurent Gbagbo aux valeurs morales et nationales par la construction d'un ethos de vertu, de courage, d'humanité et de 
solidarité (quand il compatit aux afflictions du public venu à ses meetings). L'autre, explicite, révélant comme morale de l'histoire contée la nécessité de la gratitude. Cependant, en vertu du principe qui veut que l'on ne parle bien d'une vertu que dans la mesure où l'on est à même de l'exemplifier, Laurent Gbagbo se définit comme un homme vertueux parce que reconnaissant à l'égard des personnages cités qui avaient assuré sa sécurité, comme un homme fidèle à de longues amitiés, bref un homme de parole et d'honneur.

L'on perçoit ainsi par quelles modalités la narration de soi peut suggérer des relents perlocutoires complexes. Le récit de soi permet de revendiquer certains épisodes de la vie du sujet comme étant fondamentaux à la construction du personnage discursif projeté en termes d'idéalité sociale. Idéalité revendiquée par le subtil recours à l'ethos. Toutefois, le locuteur-narrateur évoluant dans une intrigue est en relation avec d'autres personnages qui influent sur le cours des évènements qu'il rapporte et interagissent de facto avec lui en modelant son parcours, d'où le systématique récit de l'autre.

\section{Le récit de l'autre}

Il est le fait d'une énonciation à dominance référentielle où le locuteur-narrateur emploie les pronoms de la troisième personne pour désigner le référent, un tiers personnage participant à l'intrigue de manière essentielle. Il s'agit principalement d'adjuvants ayant permis un dénouement au récit que le locuteur-narrateur juge opportun de préciser. Dans le corpus analysé, le locuteur-narrateur recourt à ce procédé à plusieurs reprises. Nous nous limiterons ici à deux occurrences.

Gossio et moi avons maintenant, des rapports multiples. Quand nous nous sommes connus, nous étions camarades. C'étaient des rapports de militantisme. Et puis, progressivement, nous sommes devenus amis et frères. Cette relation a une histoire, parce que nous avons tous souffert, et nous souffrons tous. Mais je tiens à saluer le courage de cet homme.

Banquier de formation, Marcel Gossio était un cadre de la banque Crédit de Côte d'Ivoire". Quand cette banque a été "cassée", comme tous les autres cadres, il s'est mis à chercher un nouvel emploi dans d'autres structures bancaires. Et, puis, malheureusement pour lui, 1990 est arrivé (Ndlr : année de la réinstauration du multipartisme en Côte d'Ivoire). Car il s'est déclaré, publiquement, militant du Front Populaire Ivoirien. Mais, de 1990 à 2000, il n'a plus travaillé à cause de ses couleurs politiques. Il a souffert en silence. Il faisait son travail, en créant des coordinations, des sections, et même une association de parents d'élèves. C'est pourquoi, je ne suis pas étonné de le retrouver ici pour la pose de la première pierre d'un collège. Mais, nous allons en parler. Il a souffert en silence. Son épouse avait une petite imprimerie qui leur permettait de se nourrir. Lui-même faisait de petites affaires. Cela, jusqu'à ce que le FPI ait des Ministres dans le Gouvernement de Guéï Robert (Ndlr : chef de la junte militaire issu du coup d'État de 1999). Et c'est un des ministres, le Ministre de l'Enseignement Supérieur, Séri Bailly, qui l'a nommé Directeur du Centre National des Cuvres Universitaires.

Quelques mois après, quand j'ai été élu Président de la République, il est devenu Directeur Général du Port Autonome d'Abidjan (PAA). C'est à cause de ce parcourslà, que je vous dis que Marcel Gossio était au départ, un camarade, ensuite un ami, puis, un frère. Parce qu'on ne peut pas appeler "ami ou frère", quelqu'un qu'on n'admire pas!

(Laurent Gbagbo, samedi 12 septembre 2009) 
écit est encadré par une gradation ascendante montrant l'évolution de l'intimité entre le locuteur-narrateur et son alter ego, Marcel Gossio, dont il choisit de raconter l'histoire. Celle-ci précise notamment que s'il considérait Gossio comme un " camarade ", ce statut a progressivement évolué en celui d' " ami » et de "frère ", compte tenu du « courage » et de l'endurance dont il a fait preuve. Alors qu'il était un haut cadre, il perd son emploi à cause de la politique d'austérité économique du moment. Ensuite c'est son attachement au FPI qui lui vaut de ne pas trouver d'emploi car, comme de nombreux opposants, il est persécuté et menacé jusqu'à ce qu'un gouvernement d'union autorise le FPI au gouvernement. Ce parcours, selon le locuteurnarrateur, force l'admiration envers un homme qui n'aura point vendu sa dignité pour un emploi, ni bradé son honneur en reniant ses convictions politiques. Cet épidictique de courage et de vertu est mis en exergue en vue de susciter l'adhésion au combat du FPI pour la démocratie et la liberté en dépit de l'adversité que pourrait rencontrer tous ceux qui voudraient y sacrifier, car - et c'est peut-être là la morale de cette histoire - au bout de l'effort, pointe le succès.

Voici un second exemple de ce type de récit :

Ceci dit, je voudrais vous raconter une anecdote. En effet, quand la campagne électorale pour la Présidentielle de 1990 est arrivée, je m'étais déjà habitué à ces personnes, parce qu'elles assuraient en quelque sorte ma sécurité. Mais Guédé et Kobri étant policiers, ils ne pouvaient pas, sans l'autorisation du Directeur Général de la Police Nationale, aller assurer la sécurité d'un candidat à l'élection présidentielle. Alors, qu'est-ce qu'ils ont fait? Ils ont fait une demande normale de congés. Cela leur a été accordé. Et, c'est ainsi qu'ils ont pu me suivre dans la campagne. Malheureusement pour eux, la télévision nationale m'accompagnait dans ma tournée. C'est ainsi que le caméraman, qui ne les connaissait pas comme étant des policiers, les a filmés, à Bouaké et à Gohitafla. Comme à ces meetings, il y avait beaucoup de bousculades, ils ont dû souvent intervenir, pour me dégager. La télévision les a donc filmés. Lorsque les responsables de la Police Nationale ont vu le film, ils ont été convoqués, pour dire ce qu'ils faisaient pendant leurs congés.

On leur a montré le film en question. Mais, Kobri et Guédé ont dit que ce n'était pas eux. Kobri Rémi, par exemple, a dit qu'il s'agissait certainement de son frère, mais pas de lui [Ndlr : rires dans la foule].

Bref, c'était pour vous montrer à quel point ils m'ont aidé. Il y en a eu beaucoup d'autres. Mais, comme aujourd'hui, je suis dans le village de Guédé, c'est pourquoi, j'évoque son cas. Je suis souvent venu ici. Nous sommes toujours ensemble, aussi bien dans les moments de joie que de peine.

(Laurent Gbagbo, samedi 12 septembre 2009)

L'anecdote relatée est caractéristique de la narration discursive. Elle a non seulement l'avantage de décrisper l'atmosphère en suscitant l'hilarité générale comme un code phatique, mais elle est aussi révélatrice d'une morale explicite. La gratitude du locuteur-narrateur à l'endroit de ses amis qui, envers et contre tout, ont pris des risques pour le protéger durant les heures de persécution qu'a enduré son parti politique. Les marqueurs référentiels sont nombreux et dénotent une énonciation délocutive susceptible de mettre le locuteur-narrateur en retrait. Ce récit est un morceau épidictique louant la persévérance, le courage et la générosité des hommes qui ont eu la volonté de combattre aux côtés du locuteur-narrateur contre l'injustice du parti unique d'alors, le PDCI. Non seulement l'État ne permettait pas au FPI de bénéficier de la protection des forces de sécurité quand il organisait ses meetings, mais en plus, des agents de police en congés étaient interdits d'assurer la sécurité du locuteur-narrateur lorsque sa vie était menacée par des bousculades résultant 
d'attaques de toutes sortes parfois perpétrées par la police elle-même, comme nous avons pu le voir plus haut. Le caractère humoristique de cette anecdote ne tient toutefois qu'au statut de l'énonciateur. Ce récit est parole présidentielle, parole d'un homme qui aura su venir à bout des turpitudes décrites pour mériter les honneurs et la gloire attachés au prestige présidentiel. Autrement, le récit aurait été tragique ou d'un pathétique consternant, tant les violations des droits humains peuvent sembler y relever de la banalité. D'où la construction d'un ethos de prestige, de grandeur d'âme sans laquelle il serait impossible au locuteur-narrateur de parler sans haine de ceux qui lui ont causé maints revers et qui ont tant brimé ses proches. Ethos explicite d'humanité et de solidarité encore quand le locuteur-narrateur achève son récit d'une morale claire et sans ambages : il est vertueux de soutenir dans la peine comme dans la joie ceux qui vous ont secouru quand vous en aviez le plus besoin.

\section{Construction du consensus et récits ethotiques}

L'ethos construit par le moyen de l'énonciation une personnalité discursive. Celle-ci peut être antérieure à son discours, on parle alors d'ethos préalable ou prédiscusif qui relève avant tout de l'être au monde du sujet énonciatif, de ses discours antérieurs et de l'image que l'opinion commune donne de lui notamment à travers les médias. Elle peut aussi être discursive et relever, dans le cas qui nous occupe, de ce que le locuteurnarrateur dit de manière explicite de lui dans son récit ou implicitement de sa propre personne à travers ce qu'il pose comme valeurs explicites définissant ses compagnons d'infortune. Dans un tel contexte nous définirons l'ethos explicite ou montré comme consécutif au récit de soi et l'ethos implicite ou insinué comme étant la résultante du récit de l'autre.

Le récit autorise ainsi une célébration des valeurs morales propres au groupe où l'énonciation se déploie. Ces valeurs associées à un personnage construisent "une image de soi que le locuteur projette dans son discours afin d'emporter l'adhésion de l'auditoire " (Meizoz $2003: 243$ ) ou un ethos, comme nous venons de le voir. Cet ethos individuel entre cependant en relation avec l'ethos collectif. En effet, en s'efforçant de construire et d'incarner "l'ethos général de la société », le locuteur-narrateur entend " exprimer nos valeurs partagées, penser en termes de prémisses communes, exercer à bon escient son jugement et parler pour nous " (Brinton 1985 : 55); d'où la nécessaire liaison entre la construction ethotique et l'expression de la légitimité. Les extraits cités témoignent du fait que le locuteur-narrateur se légitime à travers son parcours, son histoire. Il légitime son statut alors même que l'opposition le déclare hostile à la démocratie et le dépeint comme "assoiffé de pouvoir " (le mandat constitutionnel du Président avait déjà expiré depuis 2005). Si ses adversaires entachent ainsi son image, c'est aux fins de le rendre illégitime aux yeux des populations. La stratégie du locuteurnarrateur, qui est de s'émanciper explicitement du dialogisme - constituant comme un impératif pour les hommes politiques, leur permettant de porter coup pour coup à leurs adversaires et de répondre à leurs attaques -, semble réussie puisqu'il ne répond qu'implicitement à ses détracteurs. Il leur répond à travers plusieurs récits et anecdotes révélant de lui l'image d'un homme qui a enduré tant de brimades et de molestations avec ses proches pour la démocratie et le multipartisme, pour la liberté d'expression et l'alternance démocratique, qu'il ne saurait se montrer favorable à l'autocratie comme ses adversaires veulent le faire croire. 
Le locuteur-narrateur parvient donc, à partir de l'évocation des faits passés, à relever les indices du consensus national, dont la démocratie et les valeurs qui y sont attachées. Toutefois, s'il semble aller de soi que la démocratie est un gain pour les ivoiriens, il se positionne comme étant à l'origine de ce gain obtenu au prix de nombreuses mortifications consécutives à une rude adversité. Le but perlocutoire ici encore est d'édifier son auditoire, de se présenter en saint démocrate en arborant, non pas par pure vantardise mais comme par nécessité, ses stigmates, lauriers arrachés au totalitarisme du parti unique.

\section{Dissymétrie du miroir épidictique}

Dans le cas évoqué du récit de l'autre, celui-ci est présenté sous un jour ennoblissant et révélant sa face positive. Il s'agit donc d'un épidictique célébrant des valeurs humaines et nationales. Le locuteur-narrateur en vient ainsi implicitement à incarner ces valeurs précisément auprès de son auditoire du fait que sa coprésence narrative ne relève jamais d'une coïncidence. En effet, il se pose toujours comme étant le sujet dont l'engagement politique a permis au personnage raconté de mettre en exergue ces qualités ou valeurs adulées. De ce fait, au principe de symétrie épidictique qui suppose qu'on ne reconnaît une qualité morale à un tiers que du moment que l'on l'incarne au préalable, le locuteur-narrateur substitue celui de dissymétrie épidictique, supposant qu'il est moralement supérieur au tiers adulé. Cet implicite repose sur un ethos prédiscursif notamment lié à la fonction de président de la République constituant l'identité de statut du locuteur-narrateur. L'implicite de la prééminence du locuteurnarrateur repose aussi sur deux constats discursifs. Le premier porte sur les taxèmes de position basse employés par le locuteur-narrateur pour désigner les personnages dont il fait l'apologie. Il s'agit par exemple de l'absence des civilités consécutive à des appellatifs essentiellement nominaux et prénominaux, là où ces derniers n'auraient pu parler de lui sans en référer au code protocolaire d'usage « le président Gbagbo... ». Ces taxèmes impliquent en effet que le statut institutionnel du locuteur-narrateur en fait un garant moral plus auguste que ses adjuvants. Le second réside en la multiplicité desdits adjuvants qui dans le discours que nous analysons sont au nombre de six (Dr Zézé, Blé Christophe, Guédé Zagahi, Kobri Rémi, Akré Lucien, Marcel Gossio). En s'identifiant à six personnages différents, le locuteur-narrateur révèle six postures ethotiques qui en font un homme porteur de bien plus de valeurs que chacun des personnages racontés pris isolément.

\section{Fonction argumentative et valeur persuasive}

\section{L'influence des circonstances}

Le discours qui tient lieu de corpus est prononcé par Laurent Gbagbo le 12 septembre 2009, à douze mois de l'élection présidentielle de 2010. Il est alors chef d'État et se rend en visite à Dribouo, petit village du centre-ouest de la Côte d'Ivoire dont il procède à l'investiture de la mutuelle des cadres et à la pose de la première pierre du collège. Le contexte politique est à la tension, compte tenu des échéances électorales qui se rapprochent à grands pas. Dans de telles circonstances, non seulement chaque discours tient lieu de discours de campagne mais aussi chaque apparition du chef de l'État est 
bénéfique à la localité visitée car il est de tradition en Afrique noire que l'exécutif concentre la réalisation des actions de développement de son projet de société quelques mois avant les élections en vue de donner aux électeurs l'illusion d'une ubiquité gouvernementale efficace. Ce subterfuge a pour conséquence d'attirer la sympathie de l'électorat qui est alors convaincu de la compétence de ses dirigeants.

Quant au contexte immédiat, il dispose davantage le public à une bonne réception du discours prononcé, car il charrie le potentiel manipulateur du genre du discours d'investiture, à savoir : (i) la combinaison de la parole institutionnelle et de la légitimité sociale du locuteur qui agit et parle ostentatoirement en tant que chef d'État mais subrepticement en tant que futur candidat aux élections; (ii) la transmission de la légitimité de celui qui prononce l'investiture à un ensemble constitué comme partie intégrante de l'auditoire, ici une mutuelle de cadres locaux pour le développement. Une telle transmission érige en principe l'inviolabilité de la légitimité du locuteur qui pour l'auditoire ne saurait souffrir la moindre contestation ; (iii) la référence au sacré par la mise en abyme du discours rituel qui dans le cas d'espèce n'est pas sans rappeler le baptême par aspersion d'eau sur le groupe investi imitant l'acte judéo-chrétien d'immersion du candidat au baptême, symbole de purification, de renaissance spirituelle et d'allégeance au pasteur incarnant la divinité; (iv) la dimension performative qui, dominant d'un point de vue macro-discursif le discours, se charge de consolider le pouvoir, l'autorité, la légitimité du locuteur, car ce qu'il dit existe par le seul fait de le dire.

Les dispositions du public sont donc toutes favorables au locuteur dans la mesure où le chef de l'État ne se déplace pas d'ordinaire ni pour l'investiture de mutuelles locales ni pour la pose de la première pierre de collèges où il a pour habitude de se faire représenter. Sa présence est donc synonyme de faveur quand l'on sait la forte consistance des enveloppes budgétaires allouées aux visites internes des présidents de la République dans les contrées subsahariennes. C'est donc face à un auditoire quasi conquis et plein de gratitude envers lui que le locuteur se présente. Il axera donc son discours sur le récit de sa rencontre avec les plus grands cadres de la localité en vue de persuader l'auditoire de ce qu'il partage avec lui un passé, une histoire commune donc, un présent énonciatif et - c'est ici le but de la manipulation - un futur où les cadres ainsi honorés par sa présence et ses morceaux épidictiques à leur endroit, s'uniront avec toute la population pour lui assurer un large avantage électoral.

\section{L'anecdote}

Comme nous l'avons rappelé ci-dessus, la force de manipulation de cette image était déjà explicitée par Longin, qui soutenait qu'elle donne : « du poids, de la magnificence, et de la force au discours » en donnant vie et forme aux choses dites de sorte que ceux qui écoutent semblent voir se dérouler sous leurs yeux le film des évènements narrés.

31 En tant qu'image principale du corpus étudié, l'anecdote est surexploitée par le locuteur-narrateur qui en oublie l'exigence générique de son discours. Il en fait, dit-il, un «témoignage » en lieu et place d'un discours d'investiture. Aussi cette disposition générique nouvelle le conduit-elle à endosser une peau de conteur. Cela explique certainement les procédés de dramatisation des récits narrés, notamment l'épenthèse «Mamaaan " qui a suscité l'hilarité, ou la double interrogation narrative à travers laquelle le locuteur-narrateur développe son récit en créant une tension narrative. 

personnelles qui fondent la renommée d'un politique. Dans ce cas d'espèce, la mise en narration amplifie volontairement les traits de caractères valorisants de la personnalité $\mathrm{du}$ locuteur-narrateur. Sadowsky et Roche remarquent ainsi que : «les histoires qui deviennent plus grandes que la vie et qui prennent des proportions mythiques sont presque toujours exagérées " (John Sadowsky \& Loïck Roche 2010 : 144) car «le but d'une histoire mythique, écrit Peg Neuhaser, est d'envoyer un message pour mettre en valeur une caractéristique ou une valeur importante» (Ibid.). Le locuteur-narrateur Laurent Gbagbo montre, à travers le discours analysé, son attachement au mythe du démocrate qu'il s'est construit durant de nombreuses années; l'histoire d'un homme épris de justice et de liberté qui aura sacrifié sa liberté propre au profit de celle de son peuple, l'histoire d'un nationaliste pour qui l'identité et les valeurs nationales sont audessus de tout. La construction du mythe ainsi élaboré se déploie de manière quasi eucharistique car le mythe personnel où sont révélés ceux des alliés qui y ont participé par sédimentation crée un nouveau mythe collectif, comme a pu le noter Salmon (2007 : 102) :

Le storytelling est donc une opération plus complexe qu'on ne pourrait le croire à première vue : il ne s'agit pas seulement de "raconter des histoires" aux salariés, de cacher la réalité d'un voile de fictions trompeuses, mais aussi de faire partager un ensemble de croyances à même de susciter l'adhésion et d'orienter le flux d'émotions, bref de créer un mythe collectif et contraignant : "Les histoires peuvent être des prisons", écrit David Boje.

\section{L'argument par l'exemple}

Le récit de vie constitue, dans le cadre de cette étude, un cas type d'argumentation par l'exemple. En effet, en présentant les personnages racontés - desquels il ne s'exclue pas - sous des jours qui les font paraitre exemplaires, le locuteur-narrateur en fait des exemples à suivre. Ils constituent donc des normes morales auxquelles doit se conformer l'auditoire, une incitation à l'imitation. Lorsqu'il entreprend d'expliciter l'argumentation par l'exemple, Perelman fait d'ailleurs une remarque susceptible de justifier cette hypothèse (Perelman $2008:$ 472-473) :

Dans bien des circonstances, l'orateur manifeste clairement son intention de présenter les faits comme des exemples; mais il n'en est pas toujours ainsi. Certaines revues américaines se plaisent à raconter la carrière de tel grand industriel, de tel homme politique ou d'une étoile de cinéma, sans en tirer 
explicitement de leçon. Ces faits sont-ils simplement une contribution à l'histoire ou à la petite histoire, servent-ils d'exemples pour une généralisation spontanée, sont-ce des illustrations de quelques recettes bien connues pour réussir socialement, veut-on proposer les héros de ces récits comme modèles prestigieux, et contribuer ainsi à l'éducation du public? Rien ne permet de le dire avec certitude ; probablement le récit doit-il remplir, et remplit-il effectivement, pour différentes catégories de lecteurs, tous ces rôles simultanément. utilise le récit non seulement pour mettre en avant les qualités morales de ses proches mais aussi pour inciter l'auditoire à en être l'imitateur et, tout comme ces derniers, à lui témoigner une confiance et un dévouement sans faille dans son combat politique.

\section{L'appel aux valeurs}

L'appel aux valeurs est une stratégie dont Laurent Gbagbo fait un usage constant dans les séquences narratives de ce discours. En voici deux extraits narratifs :

Vous savez, quand on vient d'un milieu de pauvre; quand on est fils de pauvre, on aime les examens et les diplômes. Parce que, si le pouvoir se transmettait comme certains le font, nous autres, nous n'aurions jamais été ce que nous sommes. Mais, c'est parce qu'on passe les diplômes, et celui qui est fort, est déclaré reçu. Odéhouri Koudou et moi, avons passé le Baccalauréat ensemble, en 1965. Et, nous savons d'où nous venons. Nous savons que nous venons de milieu pauvre. Donc, nous devrions travailler durement. Quand on a donné les résultats du Baccalauréat en 1965 et que Odéhouri a été déclaré réussi, il a crié : "Mamaaan" (Ndlr : rire dans la foule), et il s'est mis à courir dans la cour du Lycée classique d'Abidjan. Nous, autres, avons couru aussi... Et, quand, plus tard, il a réussi à son Agrégation, il m'a encore dit, "mon village" (c'est notre surnom), heureusement qu'il y a des diplômes qu'on passe, parce que dans mon domaine, la médecine, je ne serais jamais devenu quelqu'un. Mais, comme on passe les diplômes, et qu'on les passe à l'étranger, devant des professeurs africains d'autres pays, au CAMES, c'est pourquoi, nous autres, fils de pauvres, nous pouvons réussir! Sinon, j'allais croupir là, comme un simple médecin. Je voudrais saluer sa femme. Je vais le saluer, lui-même, tout à l'heure. Odéhouri, “ayo, ayoka" (Ndlr : «merci, merci beaucoup», en bété, langue locale).

La deuxième femme que je voudrais saluer, notre femme à nous, c'est Madame Zahui Philomène, l'épouse de Zahui Marcellin. C'est la fille de Zadi Nicodème, que vous connaissez, le "Bagnon", le "Nanégnon" (Ndlr: le bel homme, le sage). Zadi Nicodème n'est pas de ma génération. Mais, quand nous étions plus jeunes, nous l'avons vu. Avant mon intervention, le professeur Dédi Séri a parlé de l'histoire des "bété" ; comment ils ont vécu. Il a fait allusion à ce qui est beau. Ici, quand on veut faire de la politique, ce n'est pas du tout évident. Il ne faut pas être trop vilain. Et Zadi Nicodème était un charmant et bel homme. Mais, c'est un homme qui a connu des injustices. Et, je n'en étais pas d'accord. Zadi Nicodème était en prison à Yamoussoukro. Après coup, quand nous avons écrit des livres, nous nous sommes rendu compte qu'il avait été dénoncé, faussement, par des gens qui voulaient être députés à sa place. Je ne pouvais accepter cela! Et je l'ai écrit dans mes livres. La calomnie casse les sociétés. La délation casse les groupes. Il nous faut construire une société de qualité, avec des hommes de qualité, qui s'élèvent par leurs qualités.

La première partie révèle l'ethos de populiste de Laurent Gbagbo. Son enfance, loin des salons dorés où ont évolué ses adversaires, fut celle d'un fils de pauvre. Une enfance qui lui aura donné le goût du travail et de la persévérance certes, mais aussi son attachement à la démocratie dont il revendique être le fils car, aime-t-il à dire, celle-ci 
est le seul système politique qui permet aux hommes de condition modeste de pouvoir atteindre la plus haute magistrature nationale.

Quant au second moment, il révèle encore l'intégrité morale et démocratique du locuteur-narrateur qui aura combattu l'injustice liée au totalitarisme du parti unique, non pas avec les armes et la violence, comme il le reproche à ses adversaires accusés d'avoir commandité plusieurs tentatives de coup d'État avant de financer et d'armer une rébellion contre lui, mais à la force des idées et des mots en publiant de nombreux ouvrages sur ses différentes propositions de gouvernement et projets de société. Cette image d'intellectuel pacifiste contraste évidemment avec celle qu'il suppose à ses adversaires : des hommes sans culture démocratique épris de déstabilisations armées.

\section{L'humour}

41 Comme le notait encore Longin, Démosthène n'a pas affirmé en vain que: «le plus grand bien qui puisse nous arriver dans la vie, c'est d'être heureux ». L'expression mécanique la plus à même d'exprimer cet état de béatitude reste le rire, d'où sa force manipulatrice que tout traité de séduction et d'influence érige en arme fatale. Les anecdotes présentes dans le corpus étudié ne dérogent pas à cette norme, car elles sont si drôles que le transcripteur souligne à deux reprises des pics d'hilarité générale dans l'auditoire à travers l'indication de lecture suivante : « (Ndlr : rires dans la foule)».

Le public ainsi amusé et joyeux en oublie la solennité et la gravité dues à titre protocolaire au chef d'État. Il s'agit donc d'une manipulation réussie puisque ce moment de bonheur est révélateur de la bonne camaraderie entre le locuteurnarrateur et les personnages racontés qui par transfert métonymique est étendue à tout l'auditoire. Ce trait de familiarité participe d'ailleurs de l'ethos de populiste tant revendiqué par Laurent Gbagbo et qui constitue un indice prédiscursif de sa distance avec les codes de la retenue exigée aux hommes d'État. De ce fait, il exacerbe un tel préjugé à travers cet humour débordant.

\section{L'appel à l'émotion}

43 L'argument ethotique est de ceux qui ne s'adressent pas à la raison mais à la sensibilité de l'auditoire. Le récit de vie tel que manifesté dans notre corpus convoque les trois procédés pathémiques constituant les règles de construction du pathos (Lausberg 1960 : 257). Premièrement, le locuteur-narrateur se montre ému, se dit ému et communique par empathie son émotion à l'auditoire: sa peine, quand il évoque des souvenirs douloureux, et sa joie de constater avec du recul que les dangers rencontrés ont permis de révéler une ingéniosité souvent drolatique dont l'issue s'est avérée heureuse.

Deuxièmement, le locuteur-narrateur ne réalise pas un témoignage en l'absence des personnages racontés. Leur présence au moment de l'énonciation est un indice de la présentation des stimuli donc de la représentation directe de l'émotion. Ce procédé pathémique ouvre des perspectives tendant à confirmer à la suite de R. Boudon que «la logique des sentiments moraux " est indissociable d'" un système de raisons solides " (R. Boudon 1994 : 30). L'inclusion des individus racontés dans l'espace discursif est en effet indiciaire de l'argument par la preuve du fait que, non seulement leur présence prouve la vérité référentielle du témoignage réalisé, mais aussi qu'il ne viendrait pas à 
l'idée du locuteur-narrateur d'exposer des épisodes inexacts au sujet de la vie d'individus qui pourraient les invalider.

Enfin, le locuteur-narrateur évoque des évènements émouvants. Ce procédé pathémique est en soi propre à tout récit de vie à en croire le processus sélectif auquel est soumise la narration de soi ou d'autrui. Ces épisodes évoquent dans notre corpus le passé douloureux de l'opposant d'alors et la ténacité de l'adversité rencontrée sur son parcours politique. Les sacrifices auxquels il dit avoir consenti en vue de l'instauration d'une démocratie vraie en Côte d'Ivoire et qu'il prétend incarner au moment de son énonciation.

C'est d'ailleurs une séquence pathique et épidictique qui achève ce discours de Laurent Gbagbo :

Beaucoup de nos camarades ont été emprisonnés. Certains sont morts en prison. Les plus nombreux à être en prison sont originaires d'ici, de Guibéroua. Je voudrais leur rendre hommage. Le Chef du village de Kpogrobré, est mort en prison. C'est à cause de tous ceux-là que nous sommes là aujourd'hui. C'est pourquoi, quand il y a quelque chose sur cette terre, je me mobilise et je me déplace. J'ai compris que les Akré, Kobri, Guédé, n'étaient pas seulement qu'une excroissance, mais, ils venaient $\mathrm{du}$ plus profond de la terre de Guibéroua, qui criait son ras-le-bol, par rapport au système qui était en place. Ils n'étaient que des représentants.

Chers frères et sœurs, je vous salue. Je suis avec vous, hier, aujourd'hui et demain.

L'intérêt de cette clausule est de cristalliser le déploiement pathique à l'œuvre dans l'ensemble des passages narrés de ce discours. L'hommage établi est un lieu commun de la configuration de l'identité collective. Si le locuteur-narrateur se réclame de la lignée des précurseurs de l'opposition au régime d'Houphouët Boigny, il crée surtout dans l'esprit de ses auditeurs la certitude que son appartenance métonymique à la terre de leurs ancêtres fait de lui le porte-parole idéal auquel ils devraient donner leurs voix. La parole de promesse qui clôt le passage est riche du double poids de la preuve par les faits passés et du parallèle christique. En premier lieu, l'embrayeur temporel « hier » réactualise par feuilletage litotique les souvenirs que le personnage-narrateur aura exposés tout le long de son discours. Ensuite le circonstant temporel « aujourd'hui » qui indique dans la matérialité discursive le moment de l'énonciation en tant qu'il est indissociable du lieu et des circonstances du discours, confirme l'idée de la constance et de la fidélité du politique à ses idées, à son engagement et à ses alliés. Enfin l'assurance de cette rigueur morale garantit à l'auditoire la fiabilité du locuteur-narrateur quant à la réalisation de ses promesses pour " demain ", c'est-à-dire par glissement sémantique pour les jours futurs, voire l'avenir plus ou moins lointain. L'on remarquera l'allusion biblique à l'atemporalité de la divinité qui hors du temps et des circonstances demeure inchangée en grandeur et en puissance depuis la nuit des temps. Ce parallèle est d'autant plus saisissant que non seulement Laurent Gbagbo se définit prédiscursivement comme un chrétien proche des milieux évangéliques américains ${ }^{7}$, mais aussi et surtout dans l'emploi discursif du présent anachronique lorsqu'il affirme «Je suis avec vous, hier (...) et demain » rappelant étrangement la célèbre réplique du créateur à Moïse qui, demandant son nom, lui répondit qu'il s'appelle : « Je suis ${ }^{8} »$. 


\section{Identités idéologiques et catégorisations axiologiques de l'univers}

48

le récit discursivement encadré dans le contexte de cette étude dévoile, et non celles qu'incarne au sens propre le locuteur-narrateur ou un personnage raconté. Il conviendrait dans cette optique de retenir l'effort entrepris par le locuteur-narrateur pour susciter une reconnaissance idéologique, c'est-à-dire un appel idéologique qui joue sur l'expression sociale de besoins communs aux hommes. À partir d'un tel axe, nous avons relevé les noyaux idéologiques à l'œuvre dans notre corpus et les référents idéologiques construits par le locuteur-narrateur. Il s'agit essentiellement de deux noyaux idéologiques fondateurs d'appels idéologiques chers au locuteur-narrateur Laurent Gbagbo : d'une part l'aspiration à la liberté, de laquelle est née l'idéologie du libéralisme (Baechler 1976: 208).Celle-ci ressort des humiliations, bastonnades, intimidations et privations que le locuteur-narrateur et ses compagnons de lutte ont essuyées de la part d'un régime non démocratique. D'où l'image de combattant pour la liberté tant revendiquée par Laurent Gbagbo, qui s'est toujours défini comme étant le père du multipartisme et de l'instauration de la démocratie en Côte d'Ivoire. D'autre part l'envie, de laquelle est né le socialisme (Baechler 1976 : 227-228). Les conditions de vie si difficiles que le locuteur-narrateur aura vécu et que ses proches et lui ont partagées aussi bien au plan familial que dans ses rapports de camaraderie, le quasi dénuement dont font cas des anecdotes doxiques au sujet de certains ministres qui, nommés lors de son élection en 2000, ont dû emprunter des costumes pour sauver les apparences lors de leur prise de fonction notamment, sont autant de raisons pathiques à même de justifier le discours populiste de Laurent Gbagbo et sa verve socialiste. Il se fait d'ailleurs surnommer «l'enfant du pays» ou encore "le candidat de la Côte d'Ivoire » par opposition à son principal adversaire, Ouattara, dont il dit qu'il n'est qu'un « héritier » matériel et pseudo-politique de Félix Houphouët Boigny, et qu'il fera surnommer « l'étranger » ou encore « le candidat de l'étranger ».

Les récits de vie de manière générale construisent, comme tout récit, une catégorisation axiologique du monde. Il s'agit, à en croire Greimas, d'une quête entreprise par un sujet envers et contre des opposants hostiles à sa démarche. Ici le locuteur-narrateur, se définissant sujet d'une quête pour la garantie des libertés démocratiques et l'égalité citoyenne, expose un témoignage valorisant de six personnages adjuvants qui auront chacun joué un rôle déterminant dans son parcours d'opposant politique à Houphouët Boigny, premier président de la République de Côte d'Ivoire. Il s'agit d'un adversaire que le locuteur-narrateur ne souhaite pas prendre le risque d'attaquer, comme nous avons pu le constater, avec l'usage d'amadoueurs discursifs visant dans le cas de notre corpus à désigner «l'axe du mal » avec pour souci de ne pas heurter de sensibilités particulières. Ce besoin de pudeur trouve sa source à la fois dans une contingence morale, qui veut qu'il soit doxiquement malsain de salir la mémoire d'un défunt, et dans un pragmatisme politique visant à se présenter, à la différence de ses adversaires, comme le vrai héritier du président Houphouët, dont il dit poursuivre le combat pour l'indépendance de la Côte d'Ivoire, contrairement aux houphouétistes affichés qui n'incarnent que les valeurs négatives du père de la nation ivoirienne, notamment sa très grande fortune personnelle et sa suspecte familiarité avec la puissance coloniale qu'est la France.

Cahiers de Narratologie, 28 | 2015 


\section{Conclusion}

Le genre narratif est par définition didactique et éducatif. Les récits de vie dans le cadre d'une énonciation macro-discursive peuvent aussi bien porter sur soi que sur un tiers. L'exemple de Laurent Gbagbo, dans le discours analysé, ici conforte l'effectivité de la construction ethotique du récit discursivement enchâssé en vue de construire un consensus valorisant pour le locuteur-narrateur. La valorisation de soi passe surtout par une dissymétrie du miroir épidictique, c'est-à-dire par une sur-représentation du moi ethotique visant à éclipser l'épidictique et à en faire une autocélébration. L'on retiendra une double fonction argumentative et persuasive, notamment à travers l'argument par l'exemple et l'appel à l'émotion entre autres, comme issues perlocutoires potentielles des récits de vie. Quoiqu'ils transpirent toujours des pesanteurs liées aux identités idéologiques à l'œuvre dans la macro-structure discursive, il y est toujours question d'une démarcation entre un "axe du mal», incarné par l'adversaire, et un "axe du bien» dont se revendique invariablement le locuteur-narrateur. Le paradoxe ici étant de conjuguer, pour Laurent Gbagbo, le devoir de mémoire implicitement accusateur envers le régime d'Houphouët Boigny, et la pudeur doxique couplée au pragmatisme stratégique de ne point salir la mémoire d'un défunt président qui reste encore vivant comme symbole national vénéré par une grande partie de l'électorat. Prouesse sans doute réussie à travers une vectorisation manipulatrice des récits présentés et dont la portée perlocutoire implique aussi bien les circonstances du discours que les stratégies de l'humour débridé, du pathos et des valeurs exploitées par le locuteur-narrateur. En choisissant d'ancrer sa légitimité dans les profondeurs des turpitudes passées qu'il a endurées avec les siens, Laurent Gbagbo leste sa parole de crédibilité aux yeux de son auditoire.

\section{BIBLIOGRAPHY}

AKOPOVA, Asya, Linguistic Manipulation : Definition and Types, International Journal of Cognitive Research in Science, Engineering and Education, Vol. 1, No. 2, 2013, En ligne : www.ijcrsee.com, consulté le 21/03/2014.

BAECHLER, Jean (1976) : Qu'est-ce que l'idéologie ?, Paris : Gallimard.

BERUT, Benjamin, « Storytelling : une nouvelle propagande par le récit? », Quaderni [En ligne], 72

/ Printemps 2010, mis en ligne le 05 avril 2012, consulté le 01 janvier 2013. URL : http:// quaderni.revues.org/479.

BOUDON, Raymond (1994) : « La logique des sentiments moraux », L'Année sociologique 44, 19-51. BRINTON, Alan (1985) : « A Rhetorical View of ad Hominem », Australian Journal of Philosophy 63, $50-63$.

BRINTON, Alan (1986) : « Ethotic Argument », History of Philosophy Quarterly 3, 255-258. 
BURGER, Marcel (1999) : Identités de statut, identités de rôle, Cahiers de linguistique française $\mathrm{n}^{\circ} 21$, 35-59.

CHARAUDEAU, Patrick (2005) : Le discours politique : Les masques du pouvoir, Paris, Vuibert.

DANBLON, Emmanuelle (2002) : Rhétorique et rationalité. Essai sur l'émergence de la critique et de la persuasion, Bruxelles, Éditions de l'Université de Bruxelles.

KUYUMCUYAN, Annie (1999) : Prolégomènes à une définition du discours narratif, Cahiers de linguistique française $n^{\circ} 21,369-386$.

LAUSBERG, Heinrich (1960) : Handbuch der literarischen Rhetorik, Munich, Max Hueber.

LEFF, Michael (2009) : «Perelman, argument ad hominem et ethos rhétorique », Argumentation et Analyse du Discours [En ligne], $\mathrm{n}^{\circ} 2$ mis en ligne le 01 avril 2009. URL : http://aad.revues.org/ index213.html.

LONGIN (1995) : Traité du Sublime, Traduction de Boileau, Paris, Le Livre de Poche, Librairie Générale Française.

MEIZOZ, Jérôme (2003) : « Ethos, champ et facture des œuvres : recherches sur la "posture" ", Pratiques 117-118, 241-250.

PERELMAN, Chaïm et OLBRECHTS-TYTECA, Lucie (2008) : Traité de l'argumentation, Bruxelles, Éditions de l'université de Bruxelles.

RABATEL, Alain (2003) : Le problème du point de vue dans le texte de théâtre, Pratiques n 119/120.

SADOWSKY, John et ROCHE, Loïck (2010) : Les sept règles du storytelling, Paris, Pearson.

SALAVASTRU, Constantin, « Rationalité et manipulation, les sophismes dans le discours politique », Les cahiers de psychologie politique [En ligne], numéro 1, janvier 2002. URL : http:// lodel.irevues.inist.fr/cahierspsychologiepolitique/index.php?id=1656.

SALMON, Christian (2007) : Storytelling, la machine à fabriquer des histoires et à formater les esprits, Paris, La découverte.

SEARLE, J.R. (1969) : Les Actes de langage, Paris, Hermann, $1{ }^{\text {ère }}$ éd., Speech Acts, Cambridge, CUP.

VAN DIJK, Teun A. (2006) : Discourse and Manipulation, Discourse \& Society, Vol. 17(2) : 359-383, London, SAGE Publications.

\section{NOTES}

1. « [...] il n'est pas nécessaire au futur orateur d'avoir appris ce qui est véritablement juste, mais ce qui paraît tel à la foule chargée de décider ; non ce qui est réellement beau et bon, mais ce qui semble tel. C'est en effet la vraisemblance et non la vérité qui peut persuader ». Platon, Phèdre ou la beauté des âmes, Payot \& Cie, Paris, 1922, p. 141.

2. Michel Meyer, Questions de rhétorique: langage, raison et séduction, Le Livre de Poche, Librairie Générale Française, 1993, p. 8.

3. Notre traduction: «Manipulation is realized when the listener cannot see the speaker's covered intentions behind what is actually being said. As one of the key parameters of manipulative utterance is specific intentionality, in order to discriminate manipulation, one has to analyze such parameters as aim of verbal communication, communicative intention, reason, and motive. Manipulation is pragmatic aspect that achieves its goals without evident detection of communicative intention: the speaker wittingly chooses such form of utterance that lacks direct signals of his intentional condition. By increasing the level of inadequate perception of 
information field, manipulation widens illusionary subjective reality ». Akopova, Asya, "Linguistic Manipulation: Definition and Types", International Journal of Cognitive Research in Science, Engineering and Education, Vol. 1, No. 2, 2013, En ligne : www.ijcrsee.com, consulté le 21/03/2014.

4. Notre traduction: «Manipulation not only involves power, but specifically abuse of power, that is, domination. More specifically, manipulation implies the exercise of a form of illegitimate influence by means of discourse: manipulators make others believe or do things that are in the interest of the manipulator, and against the best interests of the manipulated ». Teun A. Van Dijk, Discourse and Manipulation, Discourse \& Society, Vol. 17(2): 359-383, London, SAGE Publications, 2006.

5. «manipulation is illegitimate in a democratic society, because it (re)produces, or may reproduce, inequality : it is in the best interests of powerful groups and speakers, and hurts the interests of less powerful groups and speakers [...] the crucial criteria are that people are being acted upon against their fully conscious will and interests, and that manipulation is in the best interests of the manipulator ", Ibid.

6. Notre traduction: "Manipulation is negative social psychological phenomenon exercising destructive effect upon an individual and the society as a whole », Akopova, Asya, "Linguistic Manipulation: Definition and Types", International Journal of Cognitive Research in Science, Engineering and Education, Vol. 1, No. 2, 2013, En ligne : www.ijcrsee.com, consulté le 21/03/2014.

7. Le COJEP (Congrès de la Jeunesse Panafricaine) de Charles Blé Goudé, leader d'opinion proche de Laurent Gbagbo, reçoit en grandes pompes le révérend américain Jesse Jackson en août 2009, soit un mois avant l'investiture de la mutuelle « Gotiwa » qui donne lieu au discours étudié.

8. «J'irai donc vers les enfants d'Israël, et je leur dirai : Le Dieu de vos pères m'envoie vers vous. Mais s'ils me demandent quel est son nom, que leur répondrai-je ? Dieu dit à Moïse : Je suis celui qui suis. Et il ajouta : c'est ainsi que tu répondras aux enfants d'Israël : Celui qui s'appelle "Je suis" m'a envoyé vers vous ». La Sainte Bible, traduction de Louis Second, Exode, chap. 3, versets 13 et 14 .

\section{ABSTRACTS}

This contribution intends to systematise life narratives as constructions bearing ethotic arguments, i.e. as constructions presenting a meliorative image of the speaker which reinforces the support of his audience to his theses. In such a context, to show the modes of the argumentative process in the autobiographic genre amounts to inquiring into the narrative genre and into its didactic potentialities as well as into the narrative structure of life narratives. Social markers, i.e. the set of values shared by the audience, which contribute to build up a consensus through life narratives, point to the dissymmetry of an epidictic mirror which discloses the ethotic patterns whereby self and other are narrated at the same time. However, the adulation of the other by the speaker is done with the expectation that the speaker will be even more adulated by this other, his interlocutive pole. The latter is indeed the object of an argumentative and a persuasive endeavour developed through a narrative process which configures both ideological identities, as claimed by the speaker-narrator, and axiological categories materialized in narrative-discursive terms. Our case study is former President of Côte d'Ivoire Laurent Gbagbo's inaugural address of the "Gotiwa" association on September $12^{\text {th }} 2009$. 
In this inaugural address, the clauses expressing power and the handover of power are intrinsic to the legitimation effect aimed by the speaker.

Cette contribution vise à systématiser les récits de vie comme étant des constructions porteuses d'arguments ethotiques, c'est-à-dire contribuant à présenter une image du locuteur qui soit méliorative et participe à l'adhésion de son auditoire aux thèses qu'il présente. Montrer les modalités du fonctionnement argumentatif du genre autobiographique revient, dans un tel contexte de valorisation de soi, à interroger le genre narratif et son potentiel didactique, voire manipulateur, de même que la structure narrative des récits de vie. Les marqueurs doxiques participant de la construction du consensus à travers les récits de vie, donc des valeurs communes à l'auditoire, conduisent à poser la dissymétrie du miroir épidictique comme révélateur de schèmes ethotiques où l'autre est narré en même temps que soi, mais adulé dans l'espoir de l'être encore davantage par le pôle co-énonciatif. Celui-ci, en effet, est l'objet de l'entreprise de l'argumentation et de la persuasion construite par un déploiement narratif configurant à la fois des identités idéologiques telles que le locuteur-narrateur les revendique et les catégorisations axiologiques mises en œuvre dans la matérialité narrativo-discursive. Le support choisi est un discours de Laurent Gbagbo, Président de la Côte d'Ivoire de 2000 à 2011, prononcé lors de la cérémonie d'investiture de la mutuelle "Gotiwa » le 12 septembre 2009. En tant que discours d'investiture, les clauses d'expression du pouvoir et de sa transmission y sont consubstantielles à l'effet de légitimation souhaité par le sujet locutif.

\section{INDEX}

Geographical index: Côte d'Ivoire

Keywords: life narratives, ethos, argumentation, epidictic, narrative manipulation

Mots-clés: récits de vie, ethos, argumentation, épidictique, manipulation narrative

Chronological index: XXIe siècle

\section{AUTHOR}

\section{DORGELÈS HOUESSOU}

Docteur en stylistique, linguistique et pragmatique textuelle à l'Université Alassane Ouattara de Bouaké, Côte d'Ivoire. dorgeleshouessou@yahoo.fr 